\title{
ANÁLISIS DE CONGLOMERADOS APLICADO A UNA POBLACIÓN TARDOANTIGUA EXCAVADA EN LUCENA, CÓRDOBA, ESPAÑA
}

\author{
CLUSTER ANALYSIS APPLIED TO A LATE ANTIQUITY \\ POPULATION EXCAVATED \\ IN LUCENA, CORDOBA, SPAIN
}

\author{
Juan Pablo Diéguez Ramírez ${ }^{1}$ \\ jpablodieguez@hotmail.com /https://orcid.org/0000-0002-7221-2681 \\ Daniel Botella Ortega ${ }^{1}$ \\ daniel@aytolucena.es /https://orcid.org/0000-0001-5116-0547 \\ Ricardo Ortega-Ruiz ${ }^{2}$ \\ ricardo.ortega@institutoforense.es / https://orcid.org/0000-0002-0757-8126
}

\section{RESUMEN}

La presente investigación tiene como objetivo mostrar el análisis de Conglomerados (Clusters) de la población tardoantigua de Cortijo Coracho, comparando los resultados biométricos obtenidos en ella con los que se han estimado en otras poblaciones ibéricas. El estudio histórico demuestra que es poco probable la existencia de individuos visigodos en dicha población. El objetivo del análisis comparado es clarificar dicha hipótesis inicial, comprobándose de esta forma si el mismo determina su proximidad a poblaciones hispanorromanas o bien a la población visigoda, lo cual indicaría que podrían existir individuos de ascendencia visigoda inhumados en la necrópolis investigada.

Palabras clave: Hispanorromanos; Visigodos; Análisis de conglomerados.

\footnotetext{
${ }^{1}$ Museo Arqueológico y Etnológico de la ciudad de Lucena, Córdoba, España.

${ }^{2}$ Instituto de Formación Profesional en Ciencias Forenses, Londres, Inglaterra.
} 


\begin{abstract}
The present research aims to show the Clusters' Analysis of the Cortijo Coracho's late antiquity population, comparing the biometric results obtained in it with those that have been estimated in other Iberian populations. The historical study shows that the existence of Visigoth individuals in this population is unlikely. The objective of the comparative analysis is to clarify said initial hypothesis, thus verifying whether it determines its proximity to Hispano-Roman populations or to the Visigoth population, which would indicate that there could be individuals of Visigoth descent buried in the investigated necropolis.
\end{abstract}

Key words: Hispanorromans; Sisigoths; Clusters' analyses.

\title{
CONTEXTO DE LA INVESTIGACIÓN
}

Durante la época romana la ocupación del territorio lucentino (Figura 1) se realizó, de forma sistemática e intensiva, a través de un sistema agrario de villas distribuidas estratégicamente alrededor de la principal calzada romana que atraviesa el territorio lucentino: la Vía Corduba-Anticaria-Malaca (Córdoba-Antequera-Málaga), ubicada en la mitad oeste del término municipal y con dirección norte-sur (el núcleo urbano de Lucena no existía en aquellos tiempos: Figura 2). De tal periodo destacan las intensas evidencias arqueológicas de yacimientos relacionados con alfares (figlinae como testares), alcanzando un total de 24 asentamientos con esta función.

La investigación que ahora se presenta se realizó sobre restos óseos que se encuentran depositados en los fondos del Museo Arqueológico y Etnológico de Lucena (Córdoba), procedentes de una necrópolis hispanorromana de época tardoantigua que fue descubierta en el llamado "Cortijo Coracho". 


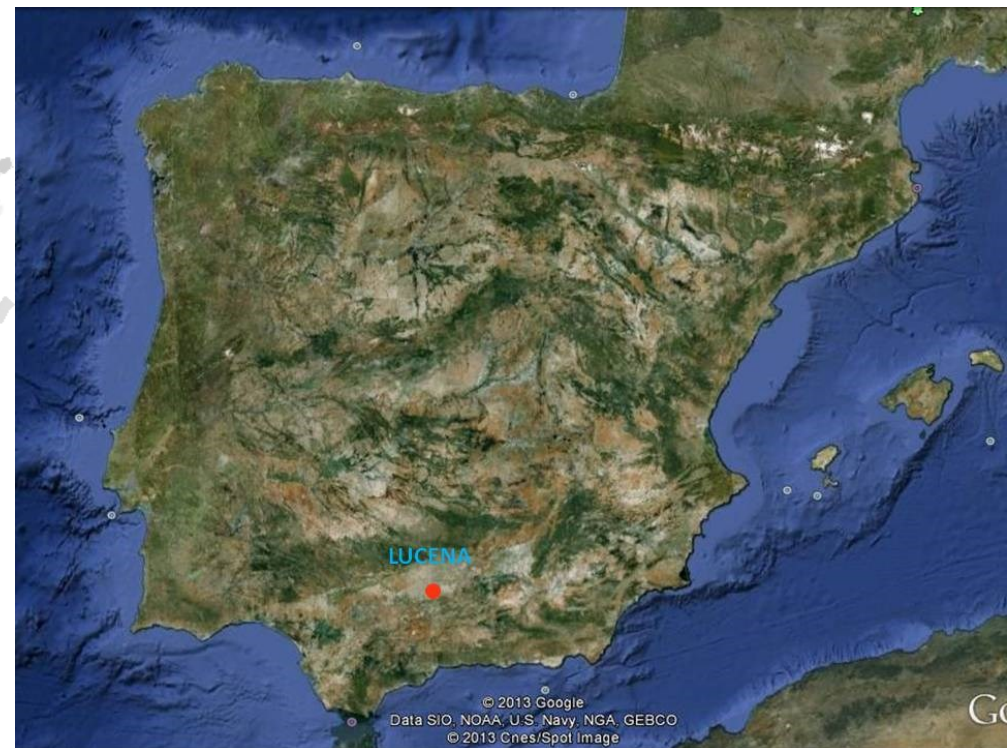

Figura 1. Localización del municipio de Lucena, (Córdoba), en la península ibérica.

La primera evidencia pública del culto del cristianismo surge en la comarca en dicho yacimiento, donde apareció una basílica martirial fechada en fase constantiniana (Figura 3). Con amortizaciones y reformas posteriores, en fase bizantina y visigoda, este complejo se completaba con una extensa necrópolis donde perviven rituales de ascendencia pagano-romana (fosas de cremación, lugares para banquetes funerarios), con ritos de inhumación de origen ya cristiano (Botella y Sánchez, 2008). 


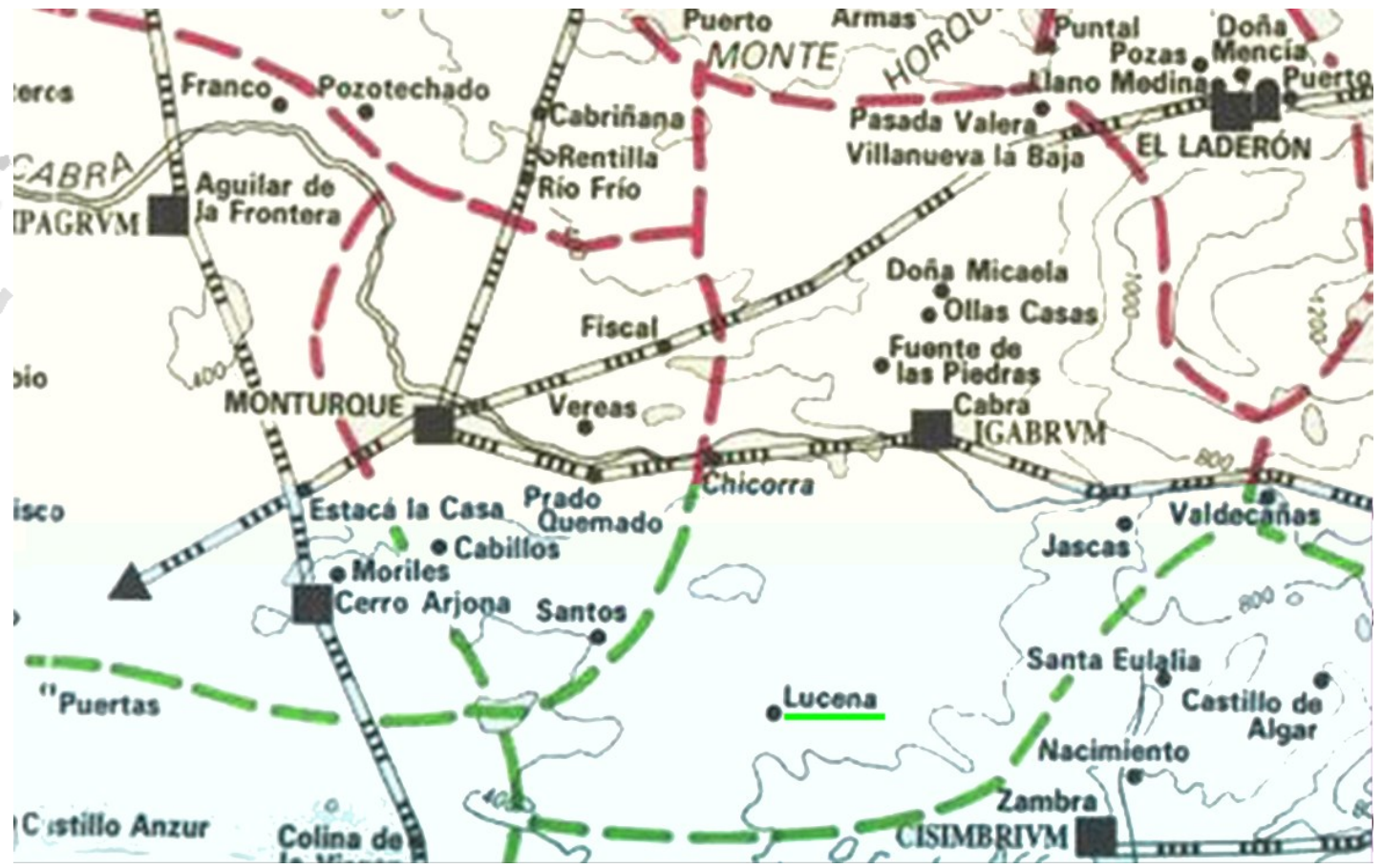

Figura 2. Mapa donde se situan las zonas habitadas durante la época romana, junto con las calzadas romanas, (el casco urbano de Lucena no existía durante esta época)

Actualmente ésta se encuentra reconstruida en el polígono industrial de la Viñuela, gracias a un convenio entre el Ayuntamiento de Lucena y el Ministerio de Fomento, que favoreció el levantamiento, casi en su totalidad, de la basílica (Figura 4). El yacimiento se excavó durante la construcción de la autovía A-45 (CórdobaMálaga), entre los años 2003-2004 (Figura 5), y se volvió a reexcavar para su traslado al casco urbano de la ciudad de Lucena. 


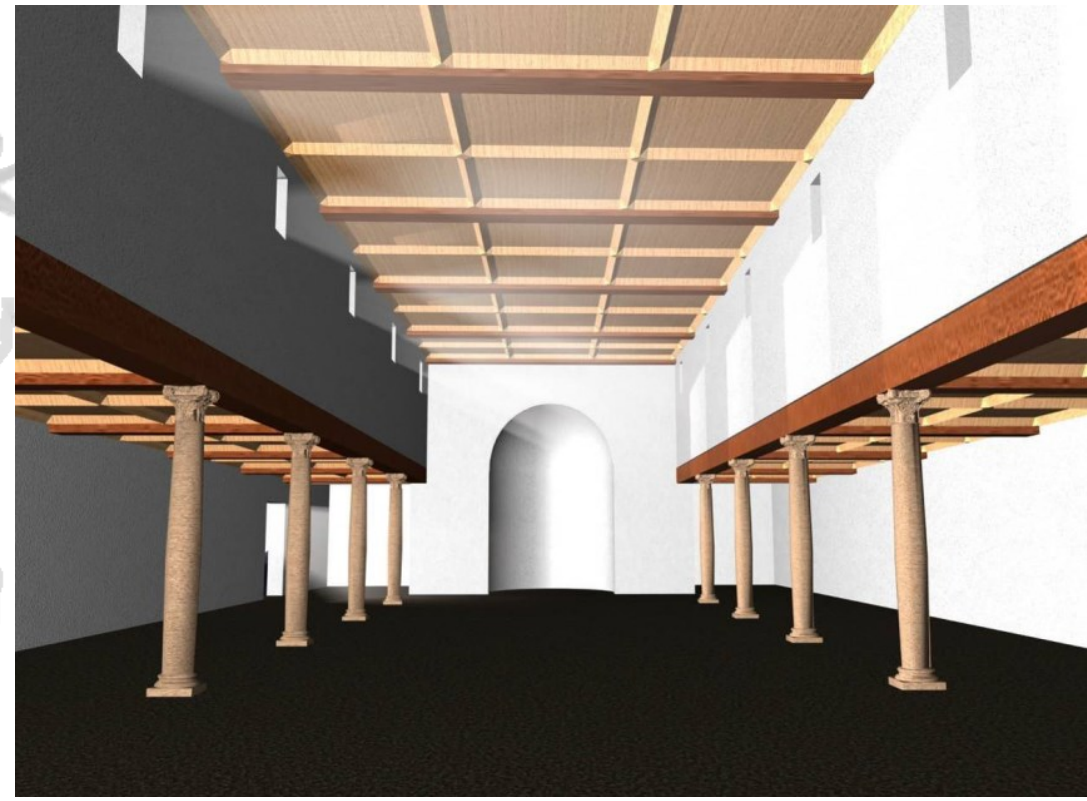

Figura 3.

Reconstrucción del interior de la Basílica.

Fase constantiniana, ( $1^{\text {a }}$ época: fase romana). 94

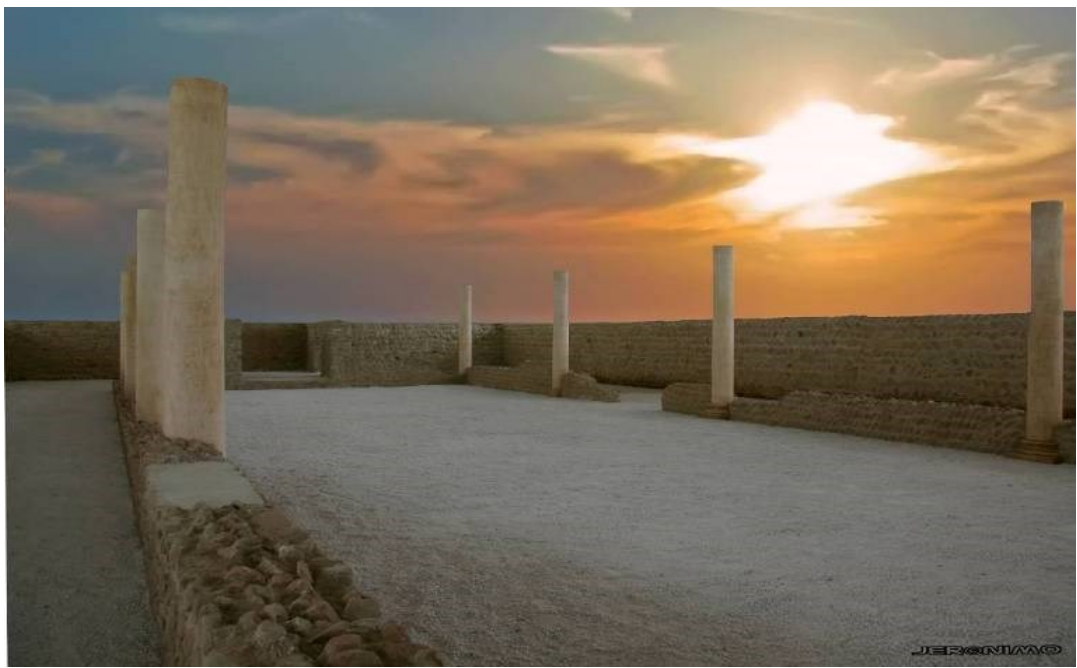

Figura 4. Vista general de la basílica reconstruida. 
La necrópolis investigada se encuentra situada en las afueras del casco urbano de Lucena (Figura 6) y la basílica asociada a ella (Botella y Sánchez, 2008) posiblemente tendría un origen previo como martyrium o memoria, en la que se depositarían los restos de algún santo o mártir de la zona (Cf. Muñiz y Bravo, 2000; Crubézy et al., 2007), aun cuando su utilidad para la evangelización de las poblaciones rurales de las villas romanas que estuviesen próximas a la misma no se descarta (Cf. Muñiz y Bravo, 2000; Díaz et al., 2007).

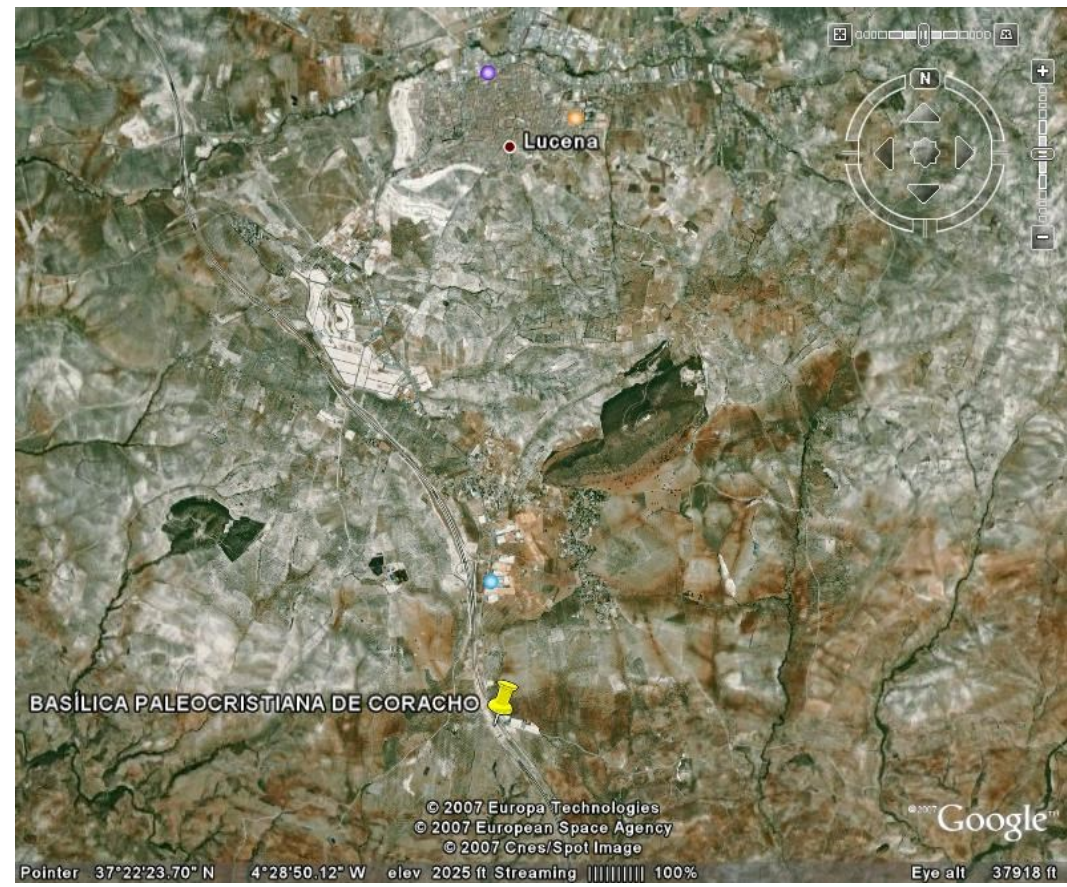

Figura 5. localización de la Basílica con referencia al municipio de Lucena, (Córdoba).

Es probable, por ello, que los restos de los individuos que allí reposaban se correspondieran con los de colonos y campesinos que trabajaban en las villas 


\section{Clio Arqueológica 2021, V36N1, p.90-127, RAMÍREZ; ORTEGA; RUIZ}

DOI: 10.51359/2448-2331.2021.250747

romanas, así como los restos de los dueños de las mismas y sus familiares, de origen hispanorromano (Cf. Carmona, 1998). La cronología de dicha necrópolis y la basílica adyacente oscila entre los siglos IV-VIII d. C. (Botella y Sánchez, 2008). La necrópolis estaba situada alrededor de la basílica, rodeándola.

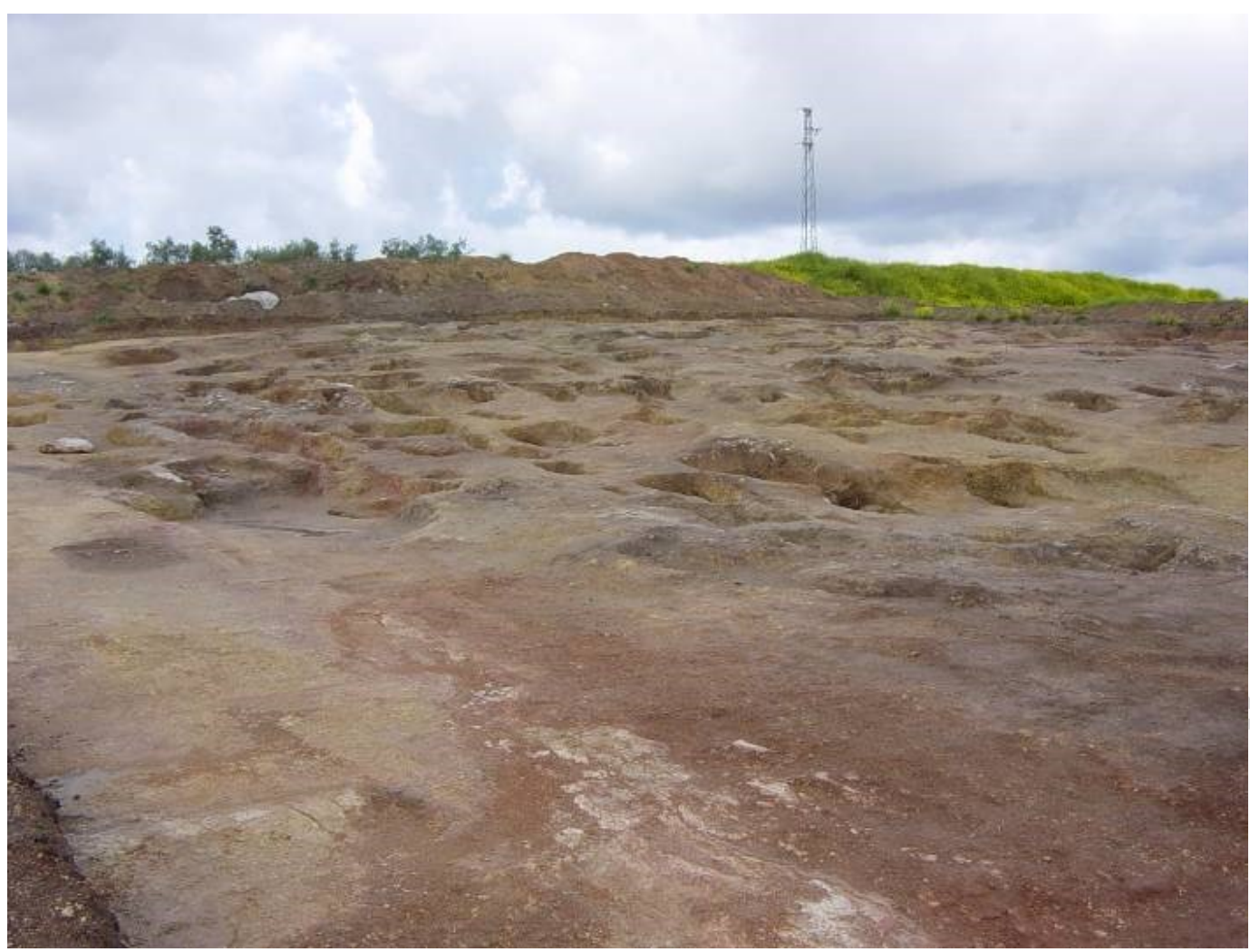

Figura 6. Vista general de la necrópolis.

Como hipótesis de trabajo, siguiendo razonamientos de tipo histórico, se considera que la población inhumada en ella se correspondería en su totalidad con población hispanorromana autóctona, asociada al grupo humano de tipo mediterráneo, 
definido este último por Campillo y Subirà (2004) o Varela (1974-75; 1973, 2015)). No obstante, de acuerdo con la cronología que se asocia a la misma, cabe suponer que quizá pudieran existir restos óseos de individuos de origen visigodo (Cf. Muñiz y Bravo, 2000; Arce, 2007; Loring et al., 2007; Jiménez, 2007).

Sin embargo, el estudio de las fuentes históricas sobre la época nos demuestra que, hasta su conquista en el año 572, por parte del rey visigodo Leovigildo, la ciudad de Corduba y la zona sur cordobesa se habían constituido como entidades independientes del poder central del reino visigodo de Toledo durante un largo periodo de tiempo, por lo que parece poco probable que existiesen restos óseos de individuos de origen visigodo con anterioridad a tales fechas (Cf. Sánchez, 2002 ; Orlandis, 2006 a y b ; Díaz et al., 2007 ; Loring et al., 2007; Thompson, 2007). De hecho, estas mismas fuentes suponen que la nobleza visigoda habitaría en las ciudades hispanas, recibiendo las rentas de las villas romanas que pasaron a ser de su propiedad sin llegar a residir en ellas (propiedad que oscilaría de un tercio a dos tercios, permaneciendo el resto de la tierra y sus rentas en manos de la nobleza hispanorromana). Por tanto, sería poco probable que fuesen enterrados en la necrópolis investigada. El presente análisis antropológico de los individuos inhumados en el yacimiento del Cortijo Coracho contrastará la veracidad de dicha hipótesis.

De acuerdo con Botella y Sánchez (2008), "teniendo en cuenta que la zona excavada de la necrópolis equivale, aproximadamente, a una sexta parte de la 
superficie sobre la que tenemos datos de la existencia de inhumaciones, y constatando la intensidad de las mismas en el único lugar donde se ha producido una intervención arqueológica (...) debemos concluir que nos encontramos ante lo que debió ser una de las mayores necrópolis conocidas de la Península para la época tardoantigua y visigoda, con una estimación aproximada mínima de más de mil tumbas. Sirva de ejemplo que la mayor necrópolis tardía excavada hasta la aparición de Coracho en la provincia de Córdoba es la de El Ruedo (Almedinilla), donde se pudieron constatar 139 tumbas". De ello se desprende la importancia del estudio de la misma, que servirá para aumentar el conocimiento de las poblaciones hispanorromanas durante la época tardoantigua.

\section{MATERIAL}

Los restos óseos se encuentran depositados en las dependencias del Museo Arqueológico y Etnológico de Lucena. Después de realizar el estudio de los mismos (Diéguez, 2015) se ha estimado un NMI (Número Mínimo de Individuos) de 397 (Tabla 1). Además, se ha comprobado que la proporción de sexos (sex ratio) es de 107:100, próxima a la habitual (105:100). 


\begin{tabular}{|c|c|c|c|c|c|c|c|c|c|}
\hline & Intervalos & & & & & Inde & minad & & \\
\hline Grupos de edad & de edad & & rones & & Iujeres & & $\begin{array}{l}\text { exo } \\
\text { nocido }\end{array}$ & & tal \\
\hline & & $N$ & $\%$ & $N$ & $\%$ & $N$ & $\%$ & $N$ & $\%$ \\
\hline Infantil I & $0-6$ & & & & & 16 & 6,35 & 16 & 4,03 \\
\hline Infantil II & $7-12$ & & & & & 31 & 12,30 & 31 & 7,81 \\
\hline Juvenil & $13-20$ & & & & & 32 & 12,70 & 32 & 8,06 \\
\hline Menores & - de 20 & & & & & 7 & 2,78 & 7 & 1,76 \\
\hline Mayores & + de 20 & 22 & 29,33 & 16 & 22,85 & 15 & 5,95 & 53 & 13,35 \\
\hline Adultos & $21-40$ & 26 & 34,66 & 38 & 54,29 & 32 & 12,70 & 96 & 24,18 \\
\hline Maduros & $41-60$ & 27 & 36,00 & 13 & 18,57 & 23 & 9,13 & 63 & 15,87 \\
\hline Seniles & + de 60 & 0 & & 3 & 4,29 & 4 & 1,59 & 7 & 1,76 \\
\hline Indeterminados & \begin{tabular}{|l|} 
Edad \\
desconocida
\end{tabular} & 0 & & 0 & & 92 & 36,50 & 92 & 23,17 \\
\hline & & 75 & 100 & 70 & 100 & 252 & 100 & 397 & 100 \\
\hline
\end{tabular}

Tabla 1. Grupos, por sexo y edad, de la población tardoantigua de Cortijo Coracho

\section{METODOLOGÍA}

\section{Determinación del sexo}

La determinación del sexo, solo investigada en adultos (Tabla 1), se ha realizado analizando, simultáneamente, cráneo y mandíbula, además de los coxales (Acsádi y Nemeskéri, 1970; Ferembach et al., 1979; Brothwell, 1993). Cuando ello no ha sido posible, o no se han obtenido datos concluyentes, se ha estimado el sexo a partir del método de Alemán et al. (1997), basado en funciones discriminantes sobre diversas variables analizadas en los huesos largos de una población mediterránea actual (de ahí el interés por su uso, ya que se trata de una población hispanorromana asociada al tipo humano mediterráneo: Alemán, 1997). Aquellos individuos en los 
que no se ha podido estimar el mismo se clasifican dentro del grupo de indeterminados (incluyendo en él a los subadultos, individuos menores de 20 años: Tabla 1).

\section{Determinación de la edad}

La estimación de la edad, en subadultos, se ha basado en el estudio de los procesos de erupción dental (Hillson, 1996; Ubelaker, 2007) y de la fusión de las epífisis de los huesos largos (Scheuer y Black, 2000; Schaefer et al., 2009). En los individuos mayores de 20 años se ha estimado analizando el desgaste dental de dientes permanentes (Guerasimov, 1955; modificado por Zoubov, 1968; citados los dos en: Krenzer, 2006; Rodríguez, 1994), empleando también el método de Lovejoy et al. (1985), para el análisis de la faceta auricular del ilion, cuando los restos óseos presentes lo han permitido.

Se utilizó la clasificación por edades de Vallois, modificada por Olivier (citado en: López, 2002): infantil I (0-6 años), infantil II (7-12 años), juvenil (13-20), en los subadultos; adultos (21-40 años), maduros (41-60 años) y seniles (más de 60 años), en los individuos mayores de 20 años de edad (Tabla 1). Aquellos individuos subadultos de edad desconocida se han incluido en un grupo denominado "menores", al igual que en el caso de individuos con más de 20 años, que se han incluido en un grupo denominado "mayores". Aquellos individuos de edad no estimada se incluyen en el grupo de indeterminados. 


\section{Caracterización biométrica de la población tardoantigua de Cortijo Coracho:}

\section{metodología}

En la investigación presente solo se ha realizado el estudio del esqueleto apendicular. Una vez identificados los huesos largos, en cada uno de los individuos estudiados, se procedió a realizar la medición de los parámetros que era posible obtener, en las condiciones en las que se encontraban dichos restos. Dichos valores son susceptibles de ser analizados con paquetes informáticos adecuados para el tratamiento estadístico de los mismos (programa SPSS V. 15), utilizando las variables definidas en Alemán (1997): varias de ellas se obtienen a partir de Martin (Martin y Saller, 1957; Martin y Knussman, 1988).

Para la obtención de estos datos se han utilizado dos pies de rey digitales, marca "Powerfix", ambos con una precisión de $0,01 \mathrm{~mm}$; 1 cinta milimetrada (con $1 \mathrm{~mm}$ de precisión) y 1 tabla osteométrica (con precisión de $1 \mathrm{~mm}$ ). Para plasmar los datos métricos obtenidos se han empleado las fichas osteológicas confeccionadas en el Laboratorio de Antropología Física de la Facultad de Medicina de la Universidad de Granada. Para la visualización de los huesos, en el Museo Arqueológico de Lucena, se ha usado una lupa binocular Motic SMZ-168, con un zoom de 0,75 x 0,5 .

\section{Objetivo de las Análisis de Conglomerados, (Cluster)}

Con los resultados obtenidos con el estudio biométrico se puede caracterizar a la población tardoantigua investigada, y se puede determinar el grado de semejanza existente entre la misma y otras poblaciones de la península ibérica: Poblaciones 
romanas (Pons), Visigodos (Varela), Poblet Iglesia, San Juan de Momoitio, Sta. María de Hito (cristiano medieval), poblaciones medievales y modernas de CastillaLeón, Cataluña medieval, la Torrecilla (musulmán medieval), Villanueva de Soportilla (cristiano medieval), Suso (cristiano medieval), Palacios Sierra (cristiano medieval), San Baudelio (cristiano medieval), Judíos de Montjuich (medieval) y Palat del Rey (medieval y moderna).

Actuar así permitiría comprobar la hipótesis de trabajo que se mantiene desde un principio: la población de Coracho es de origen hispanorromano. Sin embargo, tal vez pudiera llegar a contener individuos de ascendencia visigoda en ella. Es por ello que, de esta forma, se podría confirmar su origen o, al menos, se clarificaría su procedencia.

\section{METODOLOGÍA}

Para esta investigación se ha empleado un tipo de análisis multivariante, denominado análisis de conglomerados (cluster analysis), mediante el programa estadístico SPSS V. 15. El mismo se utiliza para agrupar elementos en grupos homogéneos, denominados conglomerados, de acuerdo con las similitudes o diferencias que presenten entre ellos, de manera que cada uno de ellos sea lo más homogéneo posible y, a la vez, que todos ellos sean muy distintos entre sí.

Los individuos, o los casos, clasificados en el mismo grupo o conglomerado (cluster), serían tan similares como fuese posible, representándose los datos 
mediante dendogramas. Se puede usar para clasificar individuos o variables. Este análisis se utiliza en Biología para clasificar animales y plantas: al mismo se le denomina "taxonomía numérica" (Bisquerra, 1989; Ferrán, 1996). Para ello se trata de conseguir que las distancias dentro de un mismo conglomerado sean pequeñas, mientras que las distancias entre conglomerados diferentes deberían de ser más amplias. Cada caso, o individuo, para cada valor de la variable estudiada, sería considerado como un punto en un espacio de "p" dimensiones (una por cada variable): se trata de unir en un mismo conglomerado los casos que sean similares entre sí, y diferentes a los que pertenezcan a otro conglomerado (Ferrán, 1996).

"Antes de iniciar un "cluster analysis" deben de tomarse tres decisiones: 1) selección de las variables relevantes para identificar a los grupos, 2) selección de la medida de proximidad entre los individiduos, 3) elección del criterio para agrupar individuos en conglomerados". (Bisquerra, 1989).

Las variables escogidas serán aquellas en las que se obtenga un mayor número de casos o poblaciones con valores observados: aparecen mencionadas para cada uno de los análisis efectuados, dentro de todas las manejadas para la presente investigación (Diéguez, 2015).

Para la selección de la medida de proximidad es conveniente estar familiarizado con este tipo de medidas, básicamente similitudes y distancias, ya que los conglomerados que se forman lo hacen en base a las proximidades entre variables o individuos. Para elegir el criterio de agrupación conviene conocer, como mínimo, 
los principales métodos de 'cluster analysis'. Se distinguen dos grandes grupos: métodos jerárquicos y no jerárquicos. Los más utilizados son los primeros, que se subdividen en aglomerativos y disociativos. Los métodos aglomerativos, o ascendentes, empiezan el análisis con tantos grupos como individuos haya, formándose grupos de forma ascendente, de manera que al final todos los casos están unidos en un mismo conglomerado. Hay muchos criterios para ir formando los conglomerados, y todos se basan en una matriz de distancias o similitudes (Bisquerra, 1989).

\begin{abstract}
"Antes de establecer el criterio para la formación de los conglomerados será necesario establecer una medida de la distancia entre individuos". La más común es la euclídea, que se define como "la raíz cuadrada de la suma de los p cuadrados de las diferencias entre los valores observados en las $p$ variables para los dos individuos correspondientes. (...) Será positiva cuando los dos individuos difieran en al menos un valor y nula cuando los dos individuos presenten exactamente los mismos valores en las $p$ variables" (Ferrán, 1996).
\end{abstract}

Una vez definida la distancia entre individuos (casos) se ha de establecer el criterio para la formación de los conglomerados. En los métodos jerárquicos aglomerativos se comienza con tantos de ellos como individuos iniciales, formándose nuevos de forma ascendente, agrupando en cada etapa a aquellos individuos que pertenezcan a los conglomerados que aparezcan más próximos, hasta que todos ellos estén, finalmente, agrupados en uno solo. Los distintos métodos difieren únicamente en la distancia considerada para medir la proximidad entre conglomerados (Ferrán, 1996). 
"El método del promedio entregrupos (average linkage between groups), también conocido como de la media, y a veces denominado UPGMA (Unweighted Pair-Group Method using aritmethic Averages), define la distancia entre dos conglomerados como el promedio de las distancias entre todos los pares de individuos en los cuales un miembro del par pertenece a cada uno de los clusters formados anteriormente. (...) Este método utiliza información de todas las distancias entre pares de individuos, y no solamente de los más alejados o de los más próximos (...). Por esta razón suele ser uno de los métodos preferidos", y es el que funciona por defecto en el SPSS V. 15 (Bisquerra, 1989). "La ventaja de este método radica en que el proceso de formación de conglomerados se puede seguir etapa a etapa. (...) Cuando el número de individuos y de variables es elevado, (...) en lugar de la propia distancia euclídea suele utilizarse su cuadrado" (Ferrán, 1996). "De la revisión de la bibliografia se deduce que, en caso de duda, el método más recomendable, probablemente, sea el del promedio entregrupos, con la distancia euclídea al cuadrado como medida de proximidad" (Bisquerra, 1989). "El uso de la distancia euclídea al cuadrado es recomendable cuando las variables vienen dadas en iguales escalas y unidades" (Maroto, 2004).

En la presente investigación se usarán el método promedio entregrupos y la medida de proximidad utilizada será la distancia euclídea al cuadrado. La representación se realizará mediante un dendograma.

\section{RESULTADOS}

Para cada extremidad y grupo de sexo se han realizado varios análisis simultáneos en los que se ha utilizado un número de variables diferente, que aparecen mencionadas en cada uno de ellos, lo cual ha permitido incluir un mayor o menor número de poblaciones en los mismos: al aumentar el número de variables investigadas se reducía el número de poblaciones ya que, en diversos casos, varias de ellas no presentaban valores para algunas de las variables analizadas. 


\section{Conglomerados para el miembro superior: sexo masculino0}

\section{Análisis I}

Para este análisis de conglomerados se han usado las siguientes medidas en huesos largos: Perímetro mínimo (en V) para el húmero, y la longitud máxima y perímetro mínimo en el radio. De un total de 17 poblaciones a estudio no se han llegado a utilizar los valores de dos de ellas $\left(\right.$ Tabla $\left.^{\circ} 2\right)$ : ver Figura 7.

Resumen de procesamiento de los $\operatorname{casos}^{a}$

\begin{tabular}{|r|r|r|r|r|r|}
\hline \multicolumn{2}{|c|}{ Casos } \\
\hline \multicolumn{2}{|c|}{ Valid } & \multicolumn{2}{c|}{ Perdidos } & \multicolumn{2}{c|}{ Total } \\
\hline $\mathrm{N}$ & Porcentaje & $\mathrm{N}$ & Porcentaje & $\mathrm{N}$ & Porcentaje \\
\hline 15 & $88,2 \%$ & 2 & $11,8 \%$ & 17 & $100,0 \%$ \\
\hline
\end{tabular}

a. Distancia euclídea al cuadrado us ada

Tabla 2. Número de poblaciones investigadas (validas), o no analizadas (casos perdidos), en el Análisis I de conglomerados para la extremidad superior en varones.

\section{Análisis II}

Para este análisis de conglomerados se han usado las siguientes medidas en huesos largos: Perímetro mínimo (en V) para el húmero, los diámetros trasversal y anteroposterior subsigmoideos en el cúbito, y la longitud máxima, perímetro mínimo y diámetros transversal y sagital en el radio. De un total de 17 poblaciones a estudio no se han llegado a utilizar los valores de ocho de ellas (Tabla 3): ver Figura 8. 
Resumen de procesamiento de los $\operatorname{casos}^{a}$

\begin{tabular}{|c|c|c|c|c|c|}
\hline \multicolumn{6}{|c|}{ Casos } \\
\hline \multicolumn{2}{|c|}{ Valid } & \multicolumn{2}{|c|}{ Perdidos } & \multicolumn{2}{|c|}{ Total } \\
\hline $\mathrm{N}$ & Porcentaje & $\mathrm{N}$ & Porcentaje & $\mathrm{N}$ & Porcentaje \\
\hline 9 & $52,9 \%$ & 8 & $47,1 \%$ & 17 & $100,0 \%$ \\
\hline
\end{tabular}

a. Distancia euclídea al cuadrado us ada

Tabla 3. Número de poblaciones investigadas (validas), o no analizadas (casos perdidos), en el Análisis II de conglomerados para la extremidad superior en varones.

Dendrogram using Average Linkage (Between Groups)

Rescaled Distance cluster Combine

Label A S E
Castilla_Lén_Mod
Cataluña Medieval
Sta Maria de Hito
villanueva de Soport
Castilla_León_Med
Suso
Poblet_Iglesia
Romanos (Pons)
visigodos (varela)
Lucena tardoantiguo
La Torrecilla
Judios Montjuich
San Juan de Momoitio
Palacios sierra
Palat del Rey

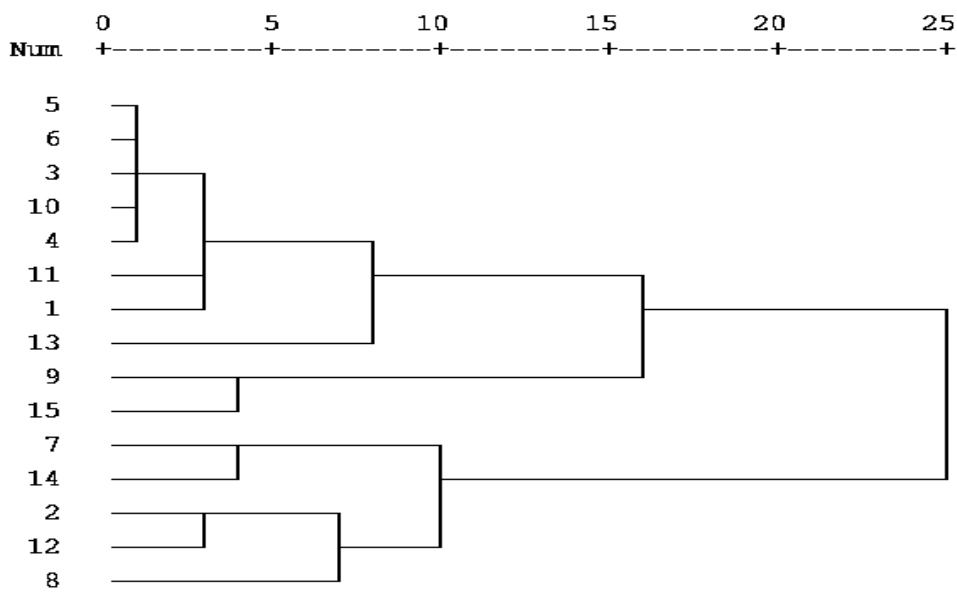

Figura 7. Dendograma obtenido tras el Análisis I de conglomerados para la extremidad superior, en varones 
Dendrogram using Average Linkage (Between Groups)

Rescaled Distance cluster Combine

Label A S E
Castilla_León_Med
Cataluña Medieval
Poblet_Iglesia
Castilla_León_Mod
Sta Maria de Hito
San Juan de Momoitio
Palat del Rey
La Torrecilla
Lucena tardoantiguo

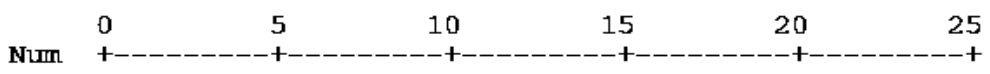

Figura 8. Dendograma obtenido tras el Análisis II de conglomerados para la extremidad superior, en varones.

\section{Conglomerados para el miembro superior: sexo femenino}

\section{Análisis I}

Para este análisis de conglomerados se han usado las siguientes medidas en huesos largos: Perímetro mínimo (en V) para el húmero, los diámetros trasversal y anteroposterior subsigmoideos en el cúbito, y el perímetro mínimo en el radio. De un total de 15 poblaciones a estudio no se han llegado a utilizar los valores de cuatro de ellas (Tabla 4): ver Figura 9. 
Resumen de procesamiento de los $\operatorname{casos}^{a}$

\begin{tabular}{|c|c|c|c|c|c|}
\hline \multicolumn{6}{|c|}{ Casos } \\
\hline \multicolumn{2}{|c|}{ Valid } & \multicolumn{2}{|c|}{ Perdidos } & \multicolumn{2}{|c|}{ Total } \\
\hline $\mathrm{N}$ & Porcentaje & $\mathrm{N}$ & Porcentaje & $\mathrm{N}$ & Porcentaje \\
\hline 11 & $73,3 \%$ & 4 & $26,7 \%$ & 15 & $100,0 \%$ \\
\hline
\end{tabular}

a. Distancia euclídea al cuadrado us ada

Tabla 4. Número de poblaciones investigadas (validas), o no analizadas (casos perdidos), en el Análisis I de conglomerados para la extremidad superior en mujeres.

\section{Análisis II}

Para este análisis de conglomerados se han usado las siguientes medidas en huesos largos: Perímetro mínimo (en V) para el húmero y el perímetro mínimo en el radio. De un total de 15 poblaciones a estudio no se han llegado a utilizar los valores de una de ellas (Tabla 5): ver Figura 10.

Resumen de procesamiento de los casos ${ }^{a}$

\begin{tabular}{|c|c|c|c|c|c|}
\hline \multicolumn{6}{|c|}{ Casos } \\
\hline \multicolumn{2}{|c|}{ Valid } & \multicolumn{2}{|c|}{ Perdidos } & \multicolumn{2}{|c|}{ Total } \\
\hline $\mathrm{N}$ & Porcentaje & $\mathrm{N}$ & Porcentaje & $\mathrm{N}$ & Porcentaje \\
\hline 14 & $93,3 \%$ & 1 & $6,7 \%$ & 15 & $100,0 \%$ \\
\hline
\end{tabular}

a. Distancia euclídea al cuadrado us ada

Tabla 5. Número de poblaciones investigadas (validas), o no analizadas (casos perdidos), en el Análisis II de conglomerados para la extremidad superior en mujeres. 
Dendrogram using Average Linkage (Between Groups)

Rescaled Distance Cluster Combine

C A S E

Label

Num

$5 \quad 10$

15

20

25

villanueva de Soport

Palacios Sierra

Cataluña Medieval

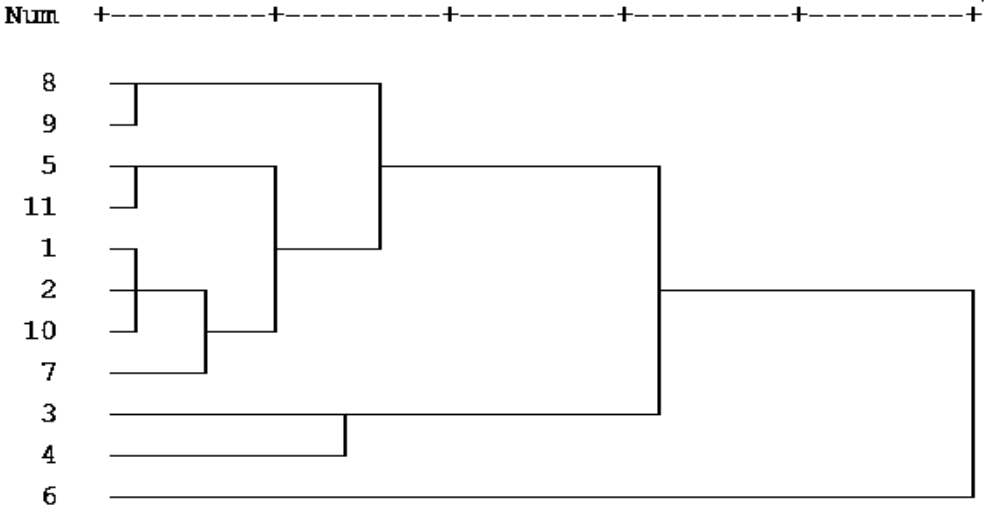

Lucena tardoantiguo

San Juan de Momoitio

Sta Maria de Hito

S_Baudelio

Palat del Rey

Castilla_León_Med

Castilla_León_Mod

La Torrecilla

Figura 9. Dendograma obtenido tras el Análisis I de conglomerados para la extremidad superior, en mujeres.

Dendrogram using Average Linkage (Between Groups)

Rescaled Distance cluster Combine

Label A S E
La Torrecilla
Judios Montjuich
villanueva de Soport
Palacios sierra
s_Baudelio
sta Maria de Hito
Romanos (Pons)
San Juan de Monoitio
Castilla_León_Mod
Palat del Rey
Castilla_León_Med
Cataluña Medieval
Iucena tardoantiguo
visigodos (Varela)

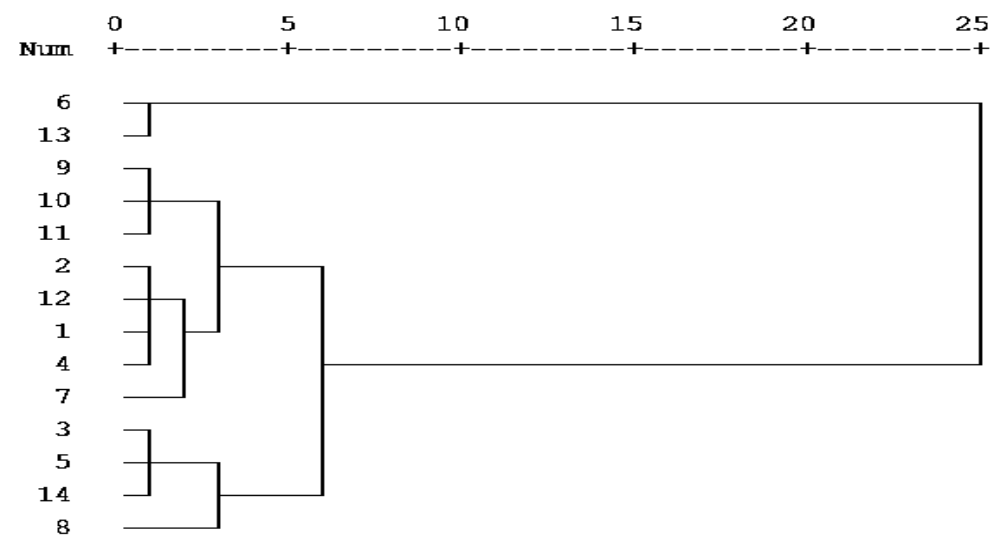

Figura 10. Dendograma obtenido tras el Análisis II de conglomerados para la extremidad superior, en mujeres. 


\section{Conglomerados para el miembro inferior: sexo masculino}

\section{Análisis I}

Para este análisis de conglomerados se han usado las siguientes medidas en huesos largos: longitud en posición y perímetro en la mitad para el fémur, y la longitud máxima, diámetros anteroposterior y transversal alrededor del agujero nutricio junto con el perímetro mínimo para la tibia. De un total de 17 poblaciones a estudio no se han llegado a utilizar los valores de nueve de ellas (Tabla 6): ver Figura 11.

Resumen de procesamiento de los casos ${ }^{a}$

\begin{tabular}{|c|c|c|c|c|c|}
\hline \multicolumn{6}{|c|}{ Casos } \\
\hline \multicolumn{2}{|c|}{ Valid } & \multicolumn{2}{|c|}{ Perdidos } & \multicolumn{2}{|c|}{ Total } \\
\hline $\mathrm{N}$ & Porcentaje & $\mathrm{N}$ & Porcentaje & $\mathrm{N}$ & Porcentaje \\
\hline 8 & $47,1 \%$ & 9 & $52,9 \%$ & 17 & $100,0 \%$ \\
\hline
\end{tabular}

a. Distancia euclídea al cuadrado us ada

Tabla 6. Número de poblaciones investigadas (validas), o no analizadas (casos perdidos), en el Análisis I de conglomerados para la extremidad inferior en varones.

\section{Análisis II}

En este análisis de conglomerados se han usado las siguientes medidas en huesos largos: longitud en posición, diámetros anteroposterior y transversal subtrocantéreos, además de los diámetros anteroposterior y transversal en la mitad y del perímetro en la mitad para el fémur, y la longitud máxima, diámetros anteroposterior y transversal alrededor del agujero nutricio junto con el perímetro mínimo para la tibia. De un total de 17 poblaciones a estudio no se han llegado a utilizar los valores de diez de ellas (Tabla 7): ver Figura 12. 
Resumen de procesamiento de los casos $^{a}$

\begin{tabular}{|r|r|r|r|r|r|}
\hline \multicolumn{9}{|c|}{ Casos } \\
\hline \multicolumn{2}{|c|}{ Valid } & \multicolumn{2}{|c|}{ Perdidos } & \multicolumn{2}{c|}{ Total } \\
\hline $\mathrm{N}$ & Porcentaje & $\mathrm{N}$ & Porcentaje & $\mathrm{N}$ & Porcentaje \\
\hline 7 & $41,2 \%$ & 10 & $58,8 \%$ & 17 & $100,0 \%$ \\
\hline
\end{tabular}

a. Distancia euclídea al cuadrado us ada

Tabla 7. Número de poblaciones investigadas (validas), o no analizadas (casos perdidos), en el Análisis II de conglomerados para la extremidad inferior en varones.

\section{Análisis III}

Resumen de procesamiento de los casos ${ }^{a}$

\begin{tabular}{|c|c|c|c|c|c|}
\hline \multicolumn{6}{|c|}{ Casos } \\
\hline \multicolumn{2}{|c|}{ Valid } & \multicolumn{2}{|c|}{ Perdidos } & \multicolumn{2}{|c|}{ Total } \\
\hline $\mathrm{N}$ & Porcentaje & $\mathrm{N}$ & Porcentaje & $\mathrm{N}$ & Porcentaje \\
\hline 13 & $76,5 \%$ & 4 & $23,5 \%$ & 17 & $100,0 \%$ \\
\hline
\end{tabular}

a. Distancia euclídea al cuadrado us ada

Tabla 8. Número de poblaciones investigadas (validas), o no analizadas (casos perdidos), en el Análisis III de conglomerados para la extremidad inferior en varones.

Se han usado las siguientes medidas en huesos largos: longitud en posición y perímetro en la mitad para el fémur, y la longitud máxima junto con el perímetro mínimo para la tibia. De un total de 17 poblaciones a estudio no se han llegado a utilizar los valores de cuatro de ellas (Tabla 8): ver Figura 13. 
Dendrogram using Average Linkage (Between Groups)

Cabel A $\mathbf{S}$
Poblet_Iglesia
Castilla_leon Med
La Torrecilla
Castilla_Leon Mod
Cataluña medieval
Suso
Lucena tardoantiguo
Sta Maria de Hito

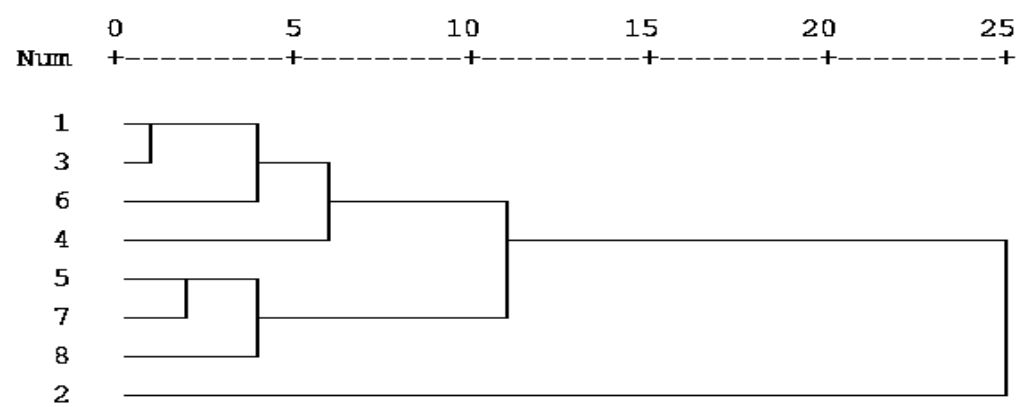

Figura 11. Dendograma obtenido tras el Análisis I de conglomerados para la extremidad inferior, en varones.

Dendrogram using Average Linkage (Between Groups)

\section{Rescaled Distance Cluster Combine}

C A S E

Label

Num 5

Poblet Iglesia Castilla_leon Med La Torrecilla Cataluña medieval Lucena tardoantiguo Castilla_Leon Mod Sta Maria de Hito

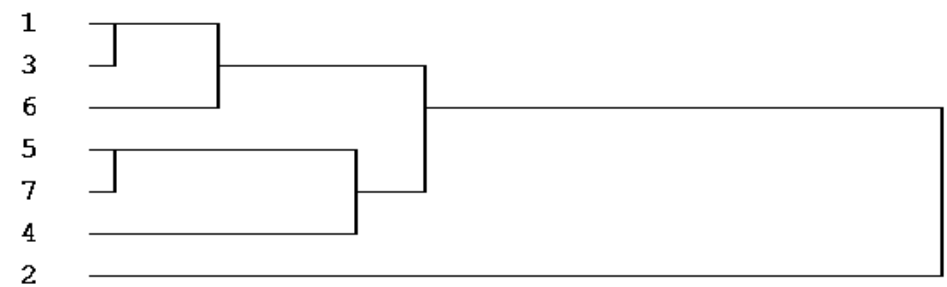

Figura 12. Dendograma obtenido tras el Análisis II de conglomerados para la extremidad inferior, en varones. 
Dendrogram using Average Linkage (Between Groups)

Rescaled Distance cluster Combine

Label

C A S E

Cataluña medieval

villanueva

Suso

Sta Maria de Hito

Castilla Leon Mod

visigodos (Varela)

Lucena tardoantiguo

Poblet_Iglesia

Castilla_leon Med

Palacios Sierra

La Torrecilla

Judios Montjuich

Romanos (Pons)

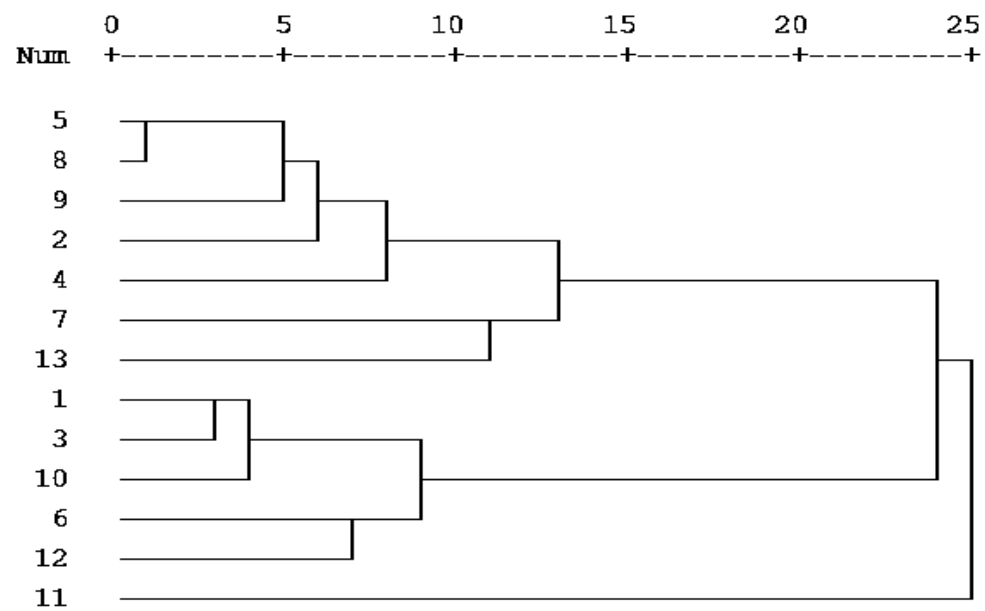

Figura 13. Dendograma obtenido tras el Análisis III de conglomerados para la extremidad inferior, en varones.

\section{Conglomerados para el miembro inferior: sexo femenino}

\section{Análisis I}

En este caso se han usado las siguientes medidas en huesos largos: longitud en posición, perímetro en la mitad, diámetros anteroposterior y transversal en la mitad y anteroposterior y transversal subtrocantéreos, para el fémur; y los diámetros anteroposterior y transversal alrededor del agujero nutricio para la tibia. De un total de 15 poblaciones a estudio no se han llegado a utilizar los valores de seis de ellas (Tabla 9): ver Figura 14. 
Resumen de procesamiento de los $\operatorname{casos}^{a}$

\begin{tabular}{|c|c|c|c|c|c|}
\hline \multicolumn{6}{|c|}{ Casos } \\
\hline \multicolumn{2}{|c|}{ Valid } & \multicolumn{2}{|c|}{ Perdidos } & \multicolumn{2}{|c|}{ Total } \\
\hline $\mathrm{N}$ & Porcentaje & $\mathrm{N}$ & Porcentaje & $\mathrm{N}$ & Porcentaje \\
\hline 9 & $60,0 \%$ & 6 & $40,0 \%$ & 15 & $100,0 \%$ \\
\hline
\end{tabular}

a. Distancia euclídea al cuadrado us ada

Tabla 9. Número de poblaciones investigadas (validas), o no analizadas (casos perdidos), en el Análisis I de conglomerados para la extremidad inferior en mujeres.

\section{Análisis II}

Resumen de procesamiento de los casos ${ }^{a}$

\begin{tabular}{|c|c|c|c|c|c|}
\hline \multicolumn{6}{|c|}{ Casos } \\
\hline \multicolumn{2}{|c|}{ Valid } & \multicolumn{2}{|c|}{ Perdidos } & \multicolumn{2}{|c|}{ Total } \\
\hline $\mathrm{N}$ & Porcentaje & $\mathrm{N}$ & Porcentaje & $\mathrm{N}$ & Porcentaje \\
\hline 12 & $80,0 \%$ & 3 & $20,0 \%$ & 15 & $100,0 \%$ \\
\hline
\end{tabular}

a. Distancia euclídea al cuadrado us ada

Tabla 10. Número de poblaciones investigadas (validas), o no analizadas (casos perdidos), en el Análisis II de conglomerados para la extremidad inferior en mujeres

Este análisis de conglomerados emplea las siguientes medidas en huesos largos: longitud en posición y perímetro en la mitad en el fémur. De un total de 15 poblaciones a estudio no se han llegado a utilizar los valores de tres de ellas (Tabla 10): ver Figura 15. 
Dendrogram using Average Linkage (Between Groups)

Rescaled Distance Cluster Combine

C A S E

Label

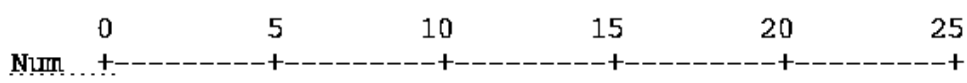

Castilla leon Med

Castilla_Leon Mod

villanueva

S_Baudelio

Cataluña medieval

Lucena tardoantiguo

Palacios Sierra

Sta Maria de Hito

La Torrecilla

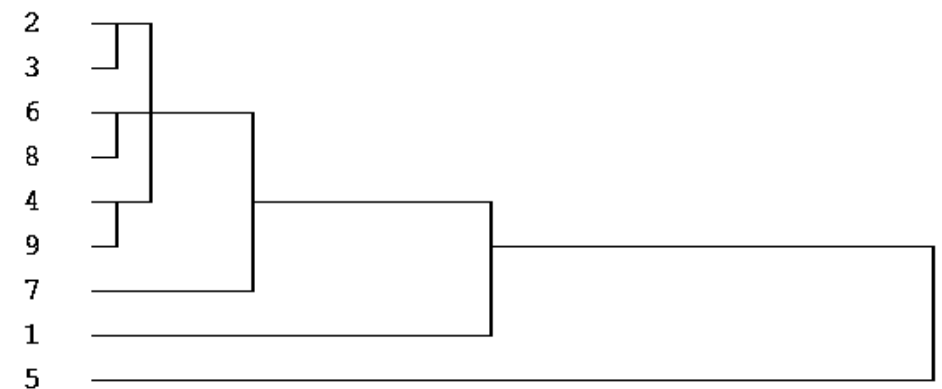

Figura 14. Dendograma obtenido tras el Análisis I de conglomerados para la extremidad inferior, en mujeres.

Dendrogram using Average Linkage (Between Groups)

Rescaled Distance cluster Combine

Label

C A S E

Castilla leon Med

villanueva

S_Baudelio

Lucena tardoantiguo

Romanos (Pons)

Sta Maria de Hito

visigodos (Varela)

Cataluña medieval

Castilla_Leon Mod

Palacios Sierra

La Torrecilla

Judios Montjuich

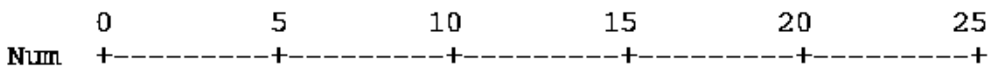

Figura 15. Dendograma obtenido tras el Análisis II de conglomerados para la extremidad inferior, en mujeres. 


\section{DISCUSIÓN}

\section{Varones}

Tras el estudio del dendograma que se obtiene en el Análisis I, para la extremidad superior de los varones, se ha comprobado (Figura 7) cómo la población masculina de la necrópolis tardoantigua aparece unida a la población visigoda, constituyendo un conglomerado que forma parte, a su vez, de aquel que asocia a las necrópolis más robustas.

El dendograma propio del Análisis II (Figura 8) deja a la población tardoantigua apartada de las demás poblaciones, constituyendo el último conglomerado con todas las demás poblaciones analizadas: no se incluye aquí a la población visigoda (de Varela) ni a la población de romanos (de Pons).

Del estudio del dendograma realizado tras el Análisis I para varones, en la extremidad inferior (Figura 11), se desprende que la población tardoantigua está próxima a la población medieval de Cataluña (esta última constituye un conglomerado con la población de Suso). No incluye este análisis a la población de visigodos (de Varela) o de romanos, (de Pons).

A su vez, después de realizar el dendograma a partir del Análisis II (Figura 12), se observa como constituyen un conglomerado las dos poblaciones anteriores ya 
citadas: Cataluña medieval y Lucena tardoantigua. No incluye este análisis a la población de visigodos (de Varela) o de romanos (de Pons).

Del Análisis III, investigando el dendograma estimado (Figura 13), que incluye a la población de visigodos peninsulares, se comprueba cómo la población tardoantigua forma un conglomerado con la misma. Ésta, a su vez, constituye un conglomerado junto con otras poblaciones robustas, entre las que se encuentra la de Cataluña medieval. La población de romanos (de Pons) se une a las demás formándose así el último conglomerado.

\section{Mujeres}

Del estudio del dendrograma del Análisis I para mujeres, en la extremidad superior (Figura 9), se desprende que la población tardoantigua está más próxima a la población de la Cataluña medieval: no aparece en este análisis la población visigoda (de Varela) ni la población de romanos (de Pons). La población de La Torrecilla es la más externa y forma el último conglomerado junto con todas las demás (no aparece la población de judíos de Montjuich en este análisis).

Respecto del dendograma del Análisis II (Figura 10) se desprende que la población tardoantigua constituye un conglomerado con las poblaciones de la Cataluña medieval y la población medieval de Castilla y León, el cual se enlaza con la población visigoda, formandose así un conglomerado superior que engloba a las cuatro. Este análisis incluye a la población de romanos (de Pons). El conglomerado 
más externo lo constituyen las poblaciones de La Torrecilla y de judíos de Montjuich, las más gráciles.

Gracias al dendograma realizado tras el Análisis I para mujeres, en la extremidad inferior (Figura 14), se observa como la población tardoantigua de Lucena constituye un conglomerado con la población de la Cataluña medieval. No aparece en este análisis la población de romanos (de Pons) o la de visigodos (de Varela).

A partir del dendograma estimado tras el Análisis II (Figura 15) se observa la constitución de dos conglomerados: uno, que incluye a la población tardoantigua junto a otras poblaciones medievales del norte peninsular, y otro que incluye a la población visigoda junto con la población medieval de Cataluña y la población de Sta. $\mathrm{M}^{\mathrm{a}}$ de Hito (todas ellas de gran robustez), estando ambos conglomerados unidos a través de la población de romanos (Tarragona y Ampurias, de Pons). En este último análisis las poblaciones más externas son La Torrecilla y los judíos de Montjuich, ambas de tipo grácil.

\section{Análisis comparado para ambos sexos}

Comparando los resultados obtenidos para ambas extremidades se puede deducir que la muestra de varones de la población tardoantigua se corresponde con una población robusta, próxima a la de Cataluña medieval, entre otras, e incluso a la población visigoda peninsular, como se desprende del Análisis I de la extremidad superior (Figura 7) y el Análisis III de la extremidad inferior (Figura 13), 
constituyéndose en ambos casos como un conglomerado conjunto con dicha población visigoda. Estaría lejos de asociarse con la población romana (de Pons).

De los análisis de ambas extremidades se desprende la mayor proximidad de la muestra femenina de la población tardoantigua con otras poblaciones del norte peninsular, también robustas, en especial con la población medieval de Cataluña. No obstante, también existe una relación, si bien de menor grado de proximidad, con la población visigoda peninsular: se observa en ambas extremidades cómo se produce una unión de $2^{\circ}$ rango de proximidad con esta última población, manteniendo una mayor proximidad con la población hispanorromana (de Pons), de acuerdo con el Análisis II de la extremidad inferior (Figura 15). Se podría deducir el menor grado de robustez y, por tanto, la mayor gracilidad presente en la muestra femenina de la población tardoantigua respecto de la muestra femenina de la población visigoda.

\section{CONCLUSIONES}

Mediante el análisis comparado de conglomerados, de la población del Cortijo Coracho con otras poblaciones peninsulares, se puede inferir la existencia de una relación de proximidad con poblaciones robustas del norte peninsular (fundamentalmente, la población medieval de Cataluña), incluida la población visigoda, si bien en mayor grado respecto de la muestra de varones de la población tardoantigua: la muestra de individuos femeninos presenta una mayor gracilidad, más próxima a la de poblaciones hispanorromanas. 
Es por ello que, aunque la gracilidad de la población tardoantigua -particularmente en lo relativo a la muestra de individuos femeninos- la relaciona con poblaciones de origen hispanorromano, no se puede afirmar con rotundidad que toda la población del Cortijo Coracho fuera de origen hispanorromano autóctono, ya que la muestra de varones presenta cierta heterogeneidad en su composición, lo cual implicaría la posible existencia en la misma de individuos masculinos de tipo robusto, menos gráciles que los de origen hispanorromano, que tal vez sí pudiesen tener un origen visigodo.

La conclusión final es que no se puede garantizar el origen hispanorromano de toda la población tardoantigua del Cortijo Coracho, sobre todo respecto de la muestra de varones. Es por ello que la hipótesis de partida (realizada tras un exhaustivo análisis histórico de la zona), según la cual la dicha población sería totalmente hispanorromana, es rechazada, al menos con los datos actuales. Se precisaría de un análisis de conglomerados nuevo que comparase los restos craneales presentes en la población tardoantigua con aquellos de las necrópolis citadas en la investigación actual, a fin de concretar mejor la conclusión anterior. 


\section{BIBLIOGRAFÍA}

ACSÁDI, Gy. y NEMESKÉRI, J. 1970. History of Human Life and Mortality. Budapest: Adademiai Kiadó.

ALEMÁN AGUILERA, I. 1997. Determinación del sexo en restos esqueléticos. Estudio de una población mediterránea actual. Tesis Doctoral. Universidad de Granada, Dpto. Ciencias Morfológicas, Laboratorio de Antropología. Granada, España.

ALEMÁN AGUILERA, I., BOTELLA LÓPEZ, M. C. y RUIZ RODRÍGUEZ, L. 1997. "Determinación del sexo en el esqueleto postcraneal. Estudio de una población mediterránea actual". Arch Esp Morfol, vol. 2: 69-79.

https://www.researchgate.net/publication/288254339_Determinacion_del_sexo_en_el_es queleto_postcraneal_Estudio_de_una_poblacion_méditerranea_actuā

ARCE, J. 2007. Bárbaros y romanos en Hispania. 400-507 A.D. Madrid: Marcial Pons, Ediciones de Historia, S.A. $2^{\mathrm{a}}$ edición.

BISQUERRA ALZINA, R. 1989. Introducción conceptual al Análisis Multivariable. Un enfoque informático con los paquetes SPSS-S, BMDP, LISREL y SPAD. Vol. I y II. Barcelona: PPU, S.A. Colección LCT-6 (Letras, Ciencias, Técnica). $1^{\mathrm{a}}$ edición.

BOTELlA ORTEGA, D. y SÁNCHEZ VELASCO, J. 2008. "La Basílica de Coracho". Lucena: Ayuntamiento de Lucena. Delegación de Patrimonio Histórico. Colección "AlYussana, Monografías de Patrimonio Arqueológico y Etnológico", $1^{\mathrm{a}}$ edición.

https://www.academia.edu/260947/La_Bas\%C3\%ADlica_de_Coracho

BROTHWELL, D. R. 1993. Desenterrando Huesos. La excavación, tratamiento y estudio de restos del esqueleto humano. Madrid: Fondo de Cultura Económica. $1^{\mathrm{a}}$ Edición en español, 1987; $1^{\text {a }}$ Reimpresión, 1993. FCE.

CAMPILLO VALERO, D. y SUBIRÀ, M. E. 2004. Antropología Física para arqueólogos. Barcelona: Editorial Ariel, S.A. $1^{\text {a }}$ edición, febrero de 2004.

CARMONA BERENGUER, S. 1998. Mundo funerario rural en la Andalucía Tardoantigua y de época visigoda. La necrópolis de El Ruedo (Almedinilla, Córdoba). Córdoba: Ediciones de la Excma. Diputación Provincial de Córdoba. 
CRUBÉZY, E., LORANS, E., MASSOT, C., PERRIN, F. y TRANOY, L. 2007. L'Archéologie Funéraire. Paris : Editions Errante, Collection ‘Archéologiques'. 2a edición.

DE LA RUA, C. y ARENAL, I. 1990. Antropología de una población medieval vizcaína. San Juan de Momoito. Garai. Cuadernos de Sección Antropología-Etnografía, vol. 7: 5-97

DÍAZ MARTÍNEZ, P. C., MARTÍNEZ MAZA, Cl. y SANZ HUESMA, F. J. 2007. Hispania tardoantigua y visigoda. Madrid: Ediciones Istmo, S.A. Serie "Historia de España", Colección "Fundamentos", nº 181.

DIÉGUEZ RAMÍREZ, J. P. 2015. Estudio Bioantropológico comparado de tres necrópolis históricas excavadas en el Término Municipal de Lucena (Córdoba). Tesis Doctoral. Universidad de Granada, Departamento de Medicina Legal, Toxicología y Antropología Física, Facultad de Medicina. Granada, España.

https://digibug.ugr.es/handle/10481/40654

Du SOUICH, H. Ph. 1977-78. Estudio antropológico de la necrópolis medieval de La Torrecilla (Arenas del Rey, Granada). Tesis doctoral. Universidad de Granada. Granada, España.

$\mathrm{Du}$ SOUICH, H. Ph. 1979. "Estudio antropológico de la necrópolis medieval de La Torrecilla (Arenas del Rey, Granada)". Antropología y Paleoecología humana, vol. 1: 2740.

Du SOUICH, H. Ph., BOTELlA LÓPEZ, M. C. y RUIZ RODRÍGUEZ, L. 1990. "Antropología de la población medieval de Palacios Sierra (Burgos)". Bol Soc Esp Antrop Biológ, vol. 11: 117-146.

Du SOUICH, H. Ph., BOTELlA LÓPEZ, M. C. y RUIZ RODRÍGUEZ, L. 1991. "Antropología de la población medieval de Villanueva de Soportilla". Antropología y Paleoecología Humana, vol. 6: 57-83.

Du SOUICH, H. Ph., MARTIN RIVAS, E. y BOTELLA LÓPEZ, M. C. 1982. "Los restos antropológicos de la necrópolis medieval de San Baudelio de Berlanga (Berlanga de Duero, Soria)". En: Souich y Guirao (Eds.). 5 Trabajos de Antropología: 30-41. Granada. 
FEREMBACH, D., SCHWIDETZKY, I. y STLOUKAL, M. 1979. "Recommandations pour determiner l'age et le sexe sur le squelette". Bulletins et Mémoires de la Société d'Anthropologie de Paris, vol. 6, série XIII: 7-45.

https://www.persee.fr/doc/bmsap_0037-8984_1979_num_6_1_1945

FERRÁN ARANAZ, M. 1996. SPSS para Windows. Programación y Análisis Estadístico. Madrid: McGRAW-HILL/ Interamericana de España, S.A. Serie McGraw-Hill de Informática.

GALERA, V. 1989. La población medieval de Santa Maria de Hito. Aspectos paleobiodemográficos, paleopatológicos, paleoepidemiológicos y de etnogénesis. Tesis doctoral. Universidad de Alcalá de Henares. Alcalá de Henares. España [Citado en: Robledo Sanz (1998); Herrerín López (2004)].

GUERASIMOV, M. M. 1955. La reconstrucción del rostro a partir del cráneo (en ruso). Moscú: Editorial Nauta. [Citado en: Krenzer (2006); Rodríguez Cuenca (1994)].

HERRERÍN LÓPEZ, J. 2004. La maqbara de Santa Clara. Estudio de una necrópolis musulmana en Cuellar. Segovia: Caja Segovia, Obra Social y Cultural; Colección "Becas de Investigación".

HILLSON, S. W. 1996. Dental Anthropology. Cambrigde: Cambrigde University Press.

JIMÉNEZ TRIGUERO, J. Mª 2007. Aproximación al estudio de los restos óseos humanos en necrópolis de la Baetica durante la Antigüedad Tardía. Tesis de Maestría. Universidad de Granada, Facultad de Medicina, Laboratorio de Antropología. Trabajo Final de Investigación, Máster de Antropología Física, Forense y Evolución Humana. Granada. España.

KRENZER, U. 2006. Compendio de Métodos Antropológico-Forenses para la reconstrucción del perfil osteo-biológico. Tomos I a VIII. Guatemala: CAFCA, Centro de Análisis Forense y Ciencias Aplicadas, Serie de Antropología Forense, $1^{a}$ edición.

https://www.academia.edu/6108359/Compendio_de_Metodos_Antropologico_Forenses_ Udo_Krenzer

LÓPEZ MARTÍNEZ, B. 2002. Los pobladores del antiguo Reino de León: Antropometría, Paleodemografía y Paleopatología. León: Universidad de León, Secretariado de Publicaciones y Medios Audiovisuales. 
LORING, Ma . I., PÉREZ, D. y FUENTES, P. 2007. La Hispania tardorromana y visigoda. Siglos V-VIII. Madrid: Editorial Síntesis S.A., Colección "Historia de España Tercer milenio".

LOVEJOY, C. O., MEINDL, R. S., PRYZBECK, Th. R. y MENSFORTH, R. P. 1985. "Chronological Metamorphosis of the Auricular Surface of the Ilium: A New Method for the Determination of Adult Skeletal Age at Death". Am. Journal of Phys Anthrop, vol. 68: $15-28$.

https://www.researchgate.net/publication/19099544_Chronological_metamorphosis_of_t he_auricular_surface_of_the_ilium_A_new_method_for_the_determination_of_adult_ske letal_age_at_death

MAROTO BENAVIDES, R. 2004. Antropología de las poblaciones femeninas medievales del Alo Ebro y Alto Duero. Tesis doctoral. Universidad de Granada. Facultad de Medicina, Laboratorio de Antropología. Granada, España.

https://digibug.ugr.es/handle/10481/1840

MARTIN, R. y KNUSSMANN, R. 1988. Lehrbuch der Anthropologie und Humangenetik. New York: Gustav Fischer Eds.

MARTIN, R. y SALLER, K. 1957. Lehrbuch der Anthropologie. Band I. Stuttgart: Gustav Fisher Verlag.

MARTIN RIVAS, E. 1980. Estudio Antropológico de la necrópolis altomedieval del Monasterio de Suso (San Millán de la Cogolla, Logroño). Memoria de Licenciatura, Facultad de Filosofía y Letras, Sección de Geografía e Historia. Universidad de Granada. Granada, España.

MARTIN RIVAS, E. y SOUICH, H. Ph. du .1981. "Estudio antropológico de la necrópolis altomedieval del Monasterio de Suso (San Millán de la Cogolla, Logroño)”. Antropología y Paleopatología Humana, vol. 2: 3-20.

MUÑIZ JAÉN, I. y BRAVO CARRASCO, A. 2000. "La necrópolis tardorromana y visigoda de El Ruedo (Almedinilla, Córdoba): una reflexión crítica”. Antiqvitas, vol. 1112: 175-189. Córdoba. 
ORLANDIS, J. 2006 a. Historia del Reino Visigodo Español. Madrid: Ediciones RIALP, S.A. $2^{\mathrm{a}}$ edición.

ORLANDIS, J. 2006 b. La vida en España en tiempo de los godos. Madrid: Ediciones RIALP, S.A. Colección "Biblioteca del Cincuentenario".

PONS ROSELL, J. 1949. "Restos humanos procedentes de la necrópolis de época romana de Tarragona y Ampurias/Gerona”. Trab Inst Fray Bernardino de Sahagún, vol. 7: 19-206.

PRADA MARCOS, M. E. 1993. Estudio antropológico de los restos humanos medievales y modernos de la necrópolis leonesa de Palat del Rey. Tesis doctoral. Universidad de León. León, España [Citado en: Robledo Sanz (1998)].

PREVOSTI, M. y PREVOSTI A. 1951. "Restos humanos procedentes de una necrópolis judaica de Montjuich (Barcelona)". Trab Inst Fray Bernardino de Sahagún, vol. 12: 69-148.

ROBLEDO SANZ, B. 1998. Dieta, indicadores de salud y caracterización biomorfológica de la población medieval musulmana de Xarea (Vélez Rubio, Almería). Tesis Doctoral. Universidad Complutense de Madrid. Facultad de Geografía e Historia, Departamento de Prehistoria. Madrid, España.

https:/leprints.ucm.es/2499/1/T22581.pdf

RODRIGUEZ CUENCA, J. V. 1994. Introducción a la Antropología Forense: análisis e identificación de restos óseos humanos. Santa Fe de Bogotá: Anaconda Editores; Universidad Nacional de Colombia.

SANCHEZ RAMOS, I. 2002. "La incidencia del Cristianismo en el mundo funerario romano cordubense". En: D. Vaquerizo (Eds.). Espacio y uso funerarios en el Occidente Romano. Córdoba. Universidad de Córdoba, Seminario de Arqueología. Vol. I y II: 325342 .

SCHAEFER, M., BLACK, S. y SCHEUER, L. 2009. Juvenile Osteology. A Laboratory and Field Manual. San Diego: Academic Press.

SCHEUER, L. y BLACK, S. 2000. The Development of Juvenile Osteology. San Diego: Academic Press. 
THOMPSON, E. A. 2007. Los godos en España. Madrid: Alianza Editorial, S.A. Colección "Historia". $1^{\mathrm{a}}$ edición en "Área de Conocimiento: Humanidades".

UBELAKER, D H. 2007. Enterramientos humanos. Excavación, análisis, interpretación. Donosti: Sociedad de Ciencias Aranzadi. MUNIBE, suplemento n 24, Gehigarria. http://www.aranzadi.eus/fileadmin/docs/Munibe/2007001200.pdf

VARELA LÓPEZ, T. A. 1973, 2015. Estudio antropológico de los restos óseos procedentes de necrópolis visigodas de la Península Ibérica. Madrid: Universidad Complutense de Madrid. Facultad de Ciencias, Sección de Biológicas. Tesis Doctoral (tesis doctorales inéditas).

https:/leprints.ucm.es/52131/1/5306713909.pdf

VARELA LÓPEZ, T. A. 1974-75. "Estudio antropológico de los restos óseos procedentes de Necrópolis visigodas de la Península Ibérica". Trab Inst Fray Bernardino de Sahagún, vol. $17,(2-3-4): 7-157$.

VIVES, E. 1987. Contribució al coneixement dels enterraments medieval a Catalunya i regios Limitrofes. Tesis Doctoral. Universidad Autónoma de Barcelona. Barcelona, España [Citado en: Herrerín López (2004)]

ZOUBOV, A. 1968. Odontología. Metódica de las investigaciones antropológicas (en ruso). Moscú: Editorial Nauta [Citado en: Krenzer (2006); Rodríguez Cuenca (1994)]. 